\title{
ESTUDIO DE SUB-ADULTOS DE LA CUENCA DEL RÍO SAN FRANCISCO, PROVINCIA DE JUJUY, ARGENTINA (0-500 DC)
}

\author{
SUB-ADULTS RESEARCHES OF THE SAN FRANCISCO'S WATERSHED RIVER, \\ JUJUY, ARGENTINA (0-500 DC)
}

\author{
Gabriela Ortiz* , Fernanda Paz², Brian Zenteno², Soledad Zuñiga² y Luis Nieva² \\ ${ }^{I}$ Consejo Nacional Científico y Tecnológico (CONICET). Centro de Investigaciones en Ciencias Sociales Regional (CISOR). Facul- \\ tad de Humanidades y Ciencias Sociales. Universidad Nacional de Jujuy. Jujuy. Argentina \\ ${ }^{2}$ Centro Regional de Estudios Arqueológicos. Facultad de Humanidades y Ciencias Sociales. Universidad Nacional de Jujuy. Jujuy. \\ Argentina
}

PALABRAS CLAVE bioarqueología; prácticas funerarias; sub-adultos; valle de San Francisco

\begin{abstract}
RESUMEN Se presenta el análisis de una muestra de individuos subadultos, exhumados en diferentes sitios de la región del valle del río San Francisco, con cronologías que abarcan ca. 2000 hasta los ca.1500 años antes del presente. Se consideraron diferentes aspectos: estado de salud (especialmente patologías de origen metabólico), edad, marcadores epigenéticos, prácticas deformatorias y los contextos de inhumación o recuperación de los restos óseos. Se registraron escasos indicadores de estrés metabólico y solo un caso de lesiones traumáticas. También se
\end{abstract}

observaron diversas prácticas culturales entre las que se incluyen deformación craneana, scalping-descarne peri y postmortem y exposición al fuego. Las formas de inhumación muestran una diversidad importante con entierros primarios en fosa, en urnas, y entierros secundarios. Se reflexiona sobre aspectos relacionados con las condiciones del ambiente donde vivieron, y las prácticas mortuorias como reflejo del rol social de los individuos subadultos. Rev Arg Antrop Biol 20(2), 2018. doi:10.17139/ raab.2018.0020.02.01

\section{KEY WORDS bioarchaeology; funerary practices; subadults; San Francisco valley}

ABSTRACT The following paper presents the analysis of a sample of subadult individuals exhumed at different sites in the San Francisco valley region, with chronologies spanning from ca. 2000 till ca. 1500 BP. Indicators such as health (particularly, metabolic pathologies), age, epigenetic markers, deformation practices, and burial contexts or contexts of recovery of skeletal remains were considered. Diverse cultural practices were also ob-

\section{La bioarqueología de los subadultos}

Tradicionalmente, los individuos no adultos fueron obviados o escasamente estudiados en lo que respecta a poblaciones osteológicas (Halcrow y Tayles, 2011; Nájera Colino et al., 2010). El menor grado de preservación respecto a los sujetos adultos y la dificultad para reconocerlos durante el proceso de exhumación por personas no familiarizadas con la anatomía de individuos inmaduros, ocasionaron que en muchas excavaciones arqueológicas no se recogieran los esqueletos de sub-adultos (Nájera Colino et al., 2010). En la última década especialmente, esta tendencia se ha revertido y cada vez existen más trabajos enfocados en su estudio. Aspectos relacionados a su crecimiento y desarrollo constituyen una excelente fuente de información sobre el entorno social y económico en el que vi- served, including craneal deformation, peri and postmortem defleshing-scalping, and exposure of the bodies to fire. Burial types showed a great diversity, with primary burials, urn burials, and secondary burials. Aspects related to environmental living conditions and mortuary practices as reflection of the social role of sub-adults are discussed. Rev Arg Antrop Biol 20(2), 2018. doi:10.17139/ raab.2018.0020.02.01

vieron. Además de la base genética, sobre ellos influyen una amplia serie de factores ambientales (físicos y culturales), que deben ser considerados al momento de su análisis (Halcrow y Tayles, 2011; Lewis, 2007; Nájera Colino et al., 2010).

La niñez, es un concepto social y cultural, y por lo tanto difiere totalmente de la noción de edad biológica. Lo que se considera "niñez" varia transculturalmente a través del tiempo en

\footnotetext{
Financiamiento: Proyecto SECTER-UNJU [C/0175]. Programa de apoyo a la investigación (FHyCS-UNJu-2015).

*Correspondencia a: Gabriela Ortiz. CREA. Otero 262. 4600 San Salvador de Jujuy. Jujuy. Argentina. E-mail: yolatordo@ hotmail.com
}

Recibido 20 Enero 2017; aceptado 8 Agosto 2017

doi:10.17139/raab.2018.0020.02.01 
todas las sociedades. Cada una tiene pautado el momento en el cual un individuo adquiere la categoría de "adulto" y son generalmente los ritos de paso, los que marcan la transición para cada etapa de la vida. La niñez es un momento crucial debido a que es el periodo en que los individuos de cualquier grupo social adquieren habilidades y conocimientos, así como aprenden el uso de la tecnología, los sistemas de creencias, valores $\mathrm{y}$ actitudes y se forma la personalidad (Sanchez Romero, 2010).

Nuestro objetivo, es caracterizar algunos de los aspectos vinculados a las condiciones de vida, salud y prácticas funerarias de los sub-adultos de la región del valle del río San Francisco durante el período Formativo del Noroeste de Argentina (NOA). La cronología de los sitios de donde proceden los restos óseos estudiados abarca desde comienzos de la era hasta $c a .500 \mathrm{dC}$. El estudio sistemático de los conjuntos óseos, nos permite acercarnos a aquellos aspectos vinculados a la vida diaria relacionados con la reproducción vital y el entorno sanitario donde los sub-adultos se desarrollaron, atendiendo a las prácticas culturales vinculadas al momento de la muerte.

\section{El registro bioarqueológico y funerario del valle de San Francisco}

El valle del río San Francisco comprende altitudinamente la franja de los 600 a $700 \mathrm{msnm}$ y se encuentra emplazado al oriente de la provincia de Jujuy (Argentina). Se trata de un amplio valle fluvial surcado por importantes cursos de agua, algunos de régimen permanente y otros estacionales (Fig. 1). Pertenece al distrito de la selva pedemontana de la región fitogeográfica de las Yungas. La investigación arqueológica de esta región se remonta a principios del siglo XX, y en la década de 1970, las investigaciones fueron retomadas (Dougherty, 1975) para ser continuadas sistemáticamente a partir de la década de 1990 (Ortiz, 2007).

Sobre la base de las características artefactuales y emplazamientos de los sitios conocidos en el sector medio y alto del valle, todos fueron adscriptos a la llamada "tradición San Francisco" (sensu Dougherty, 1975), con una profundidad temporal superior a los mil años. Estos grupos son conocidos por poseer una de las tradiciones alfareras más tempranas del NOA. Los datos re- cabados en los últimos años indican una economía "mixta" donde recursos provenientes de la caza, recolección y pesca fueron complementados con aquellos producidos en la huerta (Ortiz y Killian Galván, 2016). Las condiciones climáticas generan un ambiente con una importante biodiversidad en donde están presenten la mayor variedad de mamíferos de Argentina, de aves, fauna herpetológica y todas las especies de peces de la macro-cuenca cuenca del Paraná-Plata.

Por su clima estacional con elevadas temperaturas estivales e índices de pluviosidad por encima de $700 \mathrm{~mm}$ anuales, proliferan en la región numerosos vectores responsables de la trasmisión de distintas enfermedades, entre ellos roedores, mosquitos (Anopheles sp., Lutzomiya $s p$.), parásitos hematófagos (Ixodidae sp., Triatoma $s p$.), y gasterópodos fluviales y terrestres.

En relación al hallazgo de restos humanos, la literatura arqueológica publicada hasta hace menos de una década, da cuenta de dos formas de inhumación; entierros de párvulos en urnas, y entierro directo de adultos (Dougherty, 1975). Con el avance de las investigaciones, se conocen al presente, diferentes modalidades de tratamientos mortuorios para las denominadas poblaciones "San Francisco" (Ortiz y Nieva, 2014 a, b; Seldes y Ortiz, 2009). Las sepulturas corresponden en general a las de tipo primario directo en tierra. Tanto adultos como sub-adultos, se depositaban dentro de fosas simples, y en algunas de ellas, se habría producido un evento de quema controlada del cuerpo como parte del ritual mortuorio (Ortiz y Nieva, 2014b). También existen algunos individuos enterrados en el interior de vasijas de cerámica (Boman, 1908; Nordenskiöld, 1903). Un importante porcentaje de esqueletos tienen marcas de corte vinculadas a diferentes acciones peri y/o postmortem (descarne, desollamiento, y extracción del cuero cabelludo [scalping]). En general, han sido observadas en entierros primarios que presentan asociación anatómica y a veces, acompañamiento mortuorio. La importante recurrencia de estas prácticas en diversos contextos inhumatorios y grupos etarios, así como el tiempo e inversión de energía requeridos para intervenir los cuerpos, nos permite pensar en acciones vinculadas con el ritual funerario y no con eventos de violencia. En relación a los sitios asignados a la Tradición San Francisco, son más frecuentes 
los enterratorios que carecen de acompañamiento mortuorio, aunque en algunas sepulturas la depositación de una sola pieza de cerámica, de cuentas de collar o pipas de fumar de cerámica, formaron parte del ritual mortuorio al ser incluidas en las tumbas.

\section{MATERIAL Y MÉTODOS}

Las muestras estudiadas provienen de cuatro sitios localizados en el fondo de valle de la cuenca del río San Francisco (Fig. 1). En dos casos, los restos esqueletales fueron recuperados en el marco de rescates fortuitos por personal no entrenado. Los restantes forman parte de las colecciones obtenidas como resultado de proyectos de investigación desarrollados por nuestro equipo (Tabla 1). El sitio Pozo de la Chola es el que presenta el mayor número de individuos exhumados debido no solo a su grado de integridad sino también por la cantidad de superficie sistemáticamente excavada $\left(108 \mathrm{~m}^{2}\right)$. Los sub-adultos representan un MNI de 13 y los adultos de 33. Arroyo Colorado y Santa Ana, cuentan con solo un individuo sub-adulto y
Aguas Negras con dos (Tabla 1). Esto configura un escenario ecléctico en lo referente a la representación de las muestras analizadas, así como a las condiciones de entierro, asociaciones contextuales y preservación. Se cuenta con tres dataciones radiocarbónicas sobre restos humanos o en muestras de carbón obtenidas de los pisos donde fueron recuperados los esqueletos de subadultos (Ortiz y Nieva, 2014b; Ortiz, Ramos y Alavar, 2017) (Tabla 1). Las fechas dan cuenta de un rango de más de 500 años a partir de las dataciones más antiguas ubicadas a comienzos de la era cristiana.

Para estimar la composición de la muestra, se procedió al remontaje, seguido del análisis distribucional de los restos óseos, las características del contexto asociado y la observación en lupa de mano y binocular de señales de alteración. Para la estimación de la edad biológica se tuvieron en cuenta el grado de sinostosis de los principales centros de osificación, medición de la longitud máxima de los huesos largos y la evaluación del proceso de formación y calcificación dental (Esponda Vila, 1994; Scheuer y Black, 2000; Ubelaker, 1999). El grado de desgaste

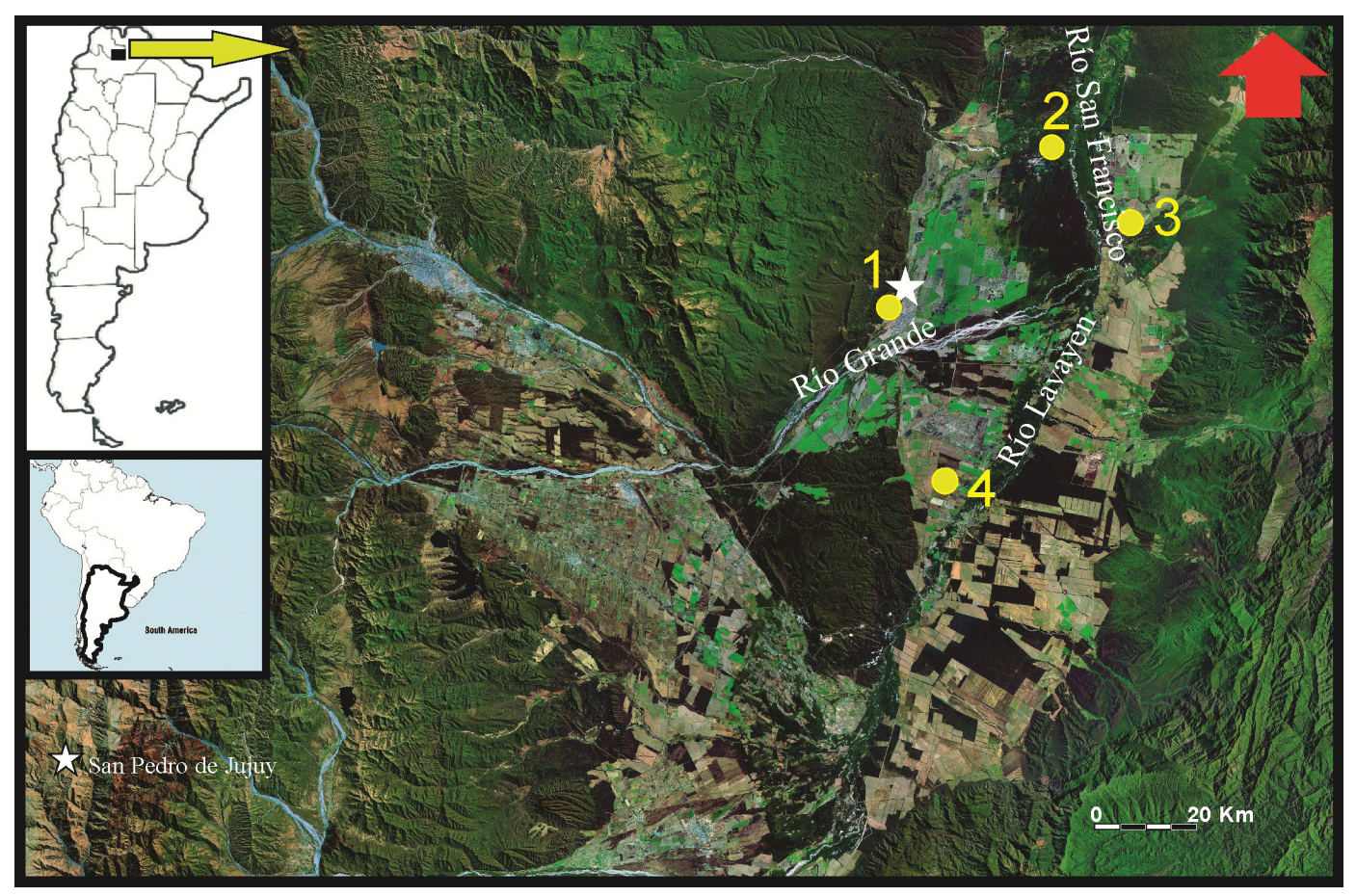

Fig. 1. Región del valle del río San Francisco con indicación de los sitios arqueológicos de donde proceden las muestras. 1. Santa Ana; 2. Pozo de la Chola; 3. Aguas Negras; 4. Arroyo Colorado 


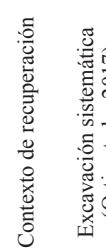

In
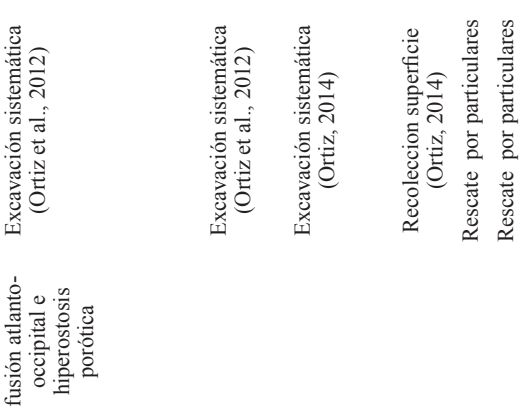

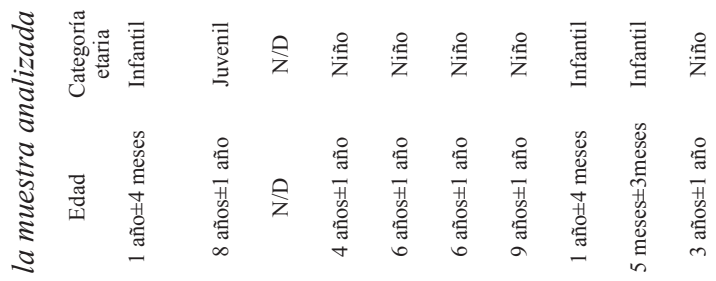

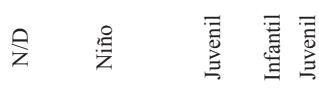

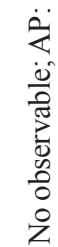

$\approx$

II

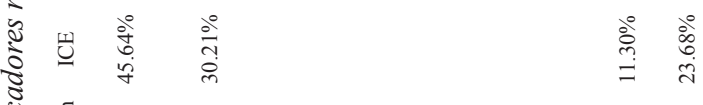

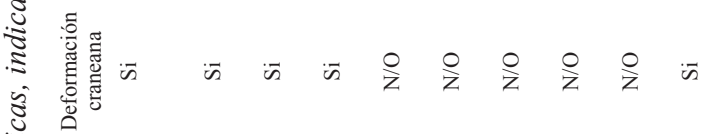

$\stackrel{1}{1}$

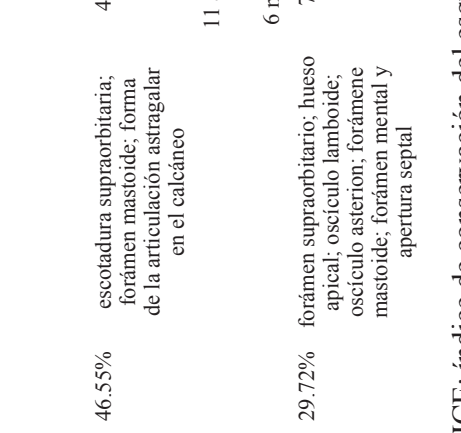

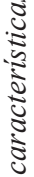

을

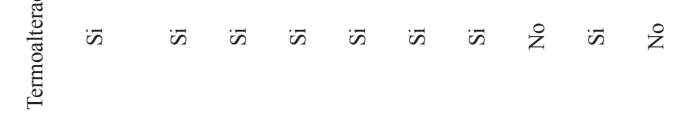

:ี

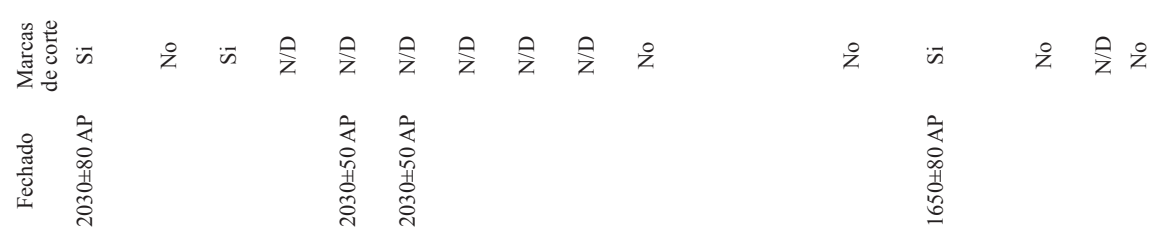

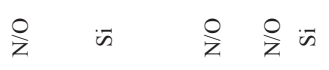

ż

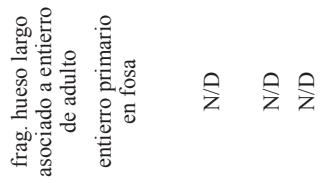

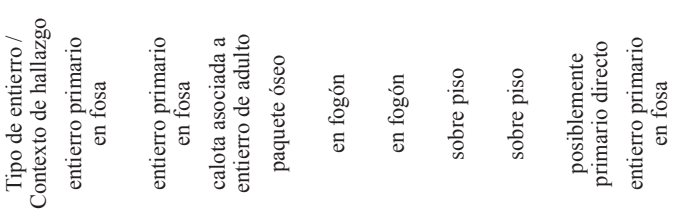

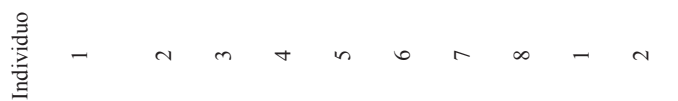

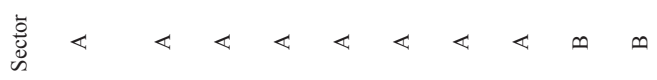

$\infty$

$\stackrel{0}{z}$

葛

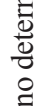

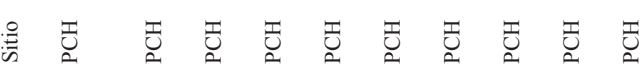

焉杂交导出

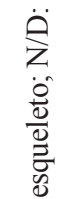

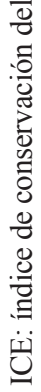

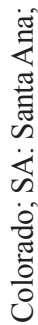

逭

悉

这 
dental, se registró mediante inspección visual siguiendo las técnicas definidas por Scott (1979) y Smith (1984) (citados en Ubelaker, 1999). La nomenclatura utilizada en la enumeración de las piezas dentales es la utilizada por la Federación Dental Internacional (FDI). Se consideraron los siguientes intervalos de edad de acuerdo a los criterios propuestos por Bogin (1988) (citado en Scheuer y Black, 2000 y Lewis, 2007): 1. Perinato: cercano al nacimiento hasta 3 meses de edad; 2. Infantil: 4 meses de edad a 2,9 años; 3 . Niño: 3 a 6,9 años y 4. Juvenil: 7 a 12,9 años. Se registraron diferentes indicadores de estrés metabólico, específicamente, criba orbitalia, hiperostosis porótica, hipoplasia del esmalte dental y líneas de Harris (Aufderheide y Rodriguez Martin, 1998; Martin, Goodman y Armelagos, 1985; Walker et al., 2009). Otra información de interés compete a los marcadores no métricos. Se registraron 31 caracteres epigenéticos en el cráneo, así como 21 del esqueleto postcraneal utilizando el método de conteo individual (Buikstra y Ubelaker, 1994). El índice de conservación del esqueleto (ICE) fue calculado de acuerdo a los criterios propuestos por Campillo y Subirá (2004) con modificaciones; ya que se calcula sobre la base del número total de huesos del cuerpo humano (a excepción de los supernumerarios, los sesamoideos y los osículos del oído medio). Debido a que el proceso de osteogénesis y los cambios histológicos a nivel esqueletal en los sub-adultos varía de acuerdo al desarrollo y la edad biológica, para calcular el índice se utilizaron las diferentes edades fisiológicas estimadas a partir del grado de madurez ósea (Meneghello, Fanta y Paris, 1972).

También se consideraron evidencias de manipulación antrópica peri y postmortem, específicamente marcas de corte e impacto siguiendo los criterios propuestos por Botella Lopez (2005); Pijoan y Pastrana (1987); Solari Giachino (2010) y Spencer (2007). Estas contemplan marcas de corte directas o indirectas, tipo de corte (por desgaste, percusión, tensión o torsión y palanqueo), localización anatómica, forma de la sección transversal, profundidad, tamaño, orientación, color, así como las acciones vinculadas con cada una de ellas (desollamiento, descarnado y desarticulado). Para el registro de la alteración térmica se observaron grados de color, localización y modificaciones de la superfi- cie externa de acuerdo a los criterios propuestos por Holck (2008); Pijoan et al. (2008) y Stodder (2008). Dos muestras de sub-adultos fueron procesadas en el INGEIS (UBA/CONICET), para estudios de paleodieta, específicamente isótopos estables para colágeno óseo $\left(\delta^{13} \mathrm{C}\right.$ y $\left.\delta^{15} \mathrm{~N}\right)$ (Tabla 1). Las distintas variedades de modificación artificial del cráneo fueron identificadas a partir de la tipología de Imbelloni (1932).

\section{Contexto de hallazgo y resultados del análisis bioarqueológico}

\section{Pozo de la Chola}

El sitio Pozo de la Chola se encuentra ubicado a la vera del cauce del río San Francisco, sobre una terraza fluvial de $2 \mathrm{~m}$ de potencia promedio en la denominada región del valle del río San Francisco (Fig. 1). El área excavada hasta el momento abarca $108 \mathrm{~m}^{2}$. Las dataciones de $\mathrm{C}^{14}$ permiten establecer una duración de la ocupación en torno al 0 de la era cristiana, hasta su posible abandono hacia el $500 \mathrm{dC}$ (Ortiz et al., 2015). La presencia de tumbas está documentada desde los inicios de la ocupación del sitio y han sido registradas en dos sectores distantes entre sí unos $50 \mathrm{~m}$ lineales. El sector A, considerado un lugar donde habrían ocurrido diversas actividades que incluyen consumo, descarte, y posiblemente preparación de alimentos; y el sector $\mathrm{B}$, interpretado como un lugar exclusivo de inhumación.

\section{Sector $A$}

Individuo 1: se trata de un entierro primario en fosa, colocado en posición decúbito dorsal extendido (Fig. 2a1). Se encontraron asociados al entierro numerosos fragmentos de carbones tanto alrededor y adentro del cráneo como en algunos sectores del cuerpo. El fechado realizado al carbón del evento de quema arrojó una edad de $2030 \pm 80$ años AP (Ortiz et al., 2017). Presenta un desgaste dental de tipo leve, ninguna carie y "dens evaginatus" en ambos $1^{\circ}$ premolares inferiores (Tabla 1 y 2). El índice de conservación es de $45,64 \%$ y el estado de preservación es bueno. En la Figura 2a2, se representan los elementos anatómicos recuperados. Considerando que el cráneo es el menos preservado solo se registró foramen mental. Presenta fusión de los cóndilos occipitales con las masas laterales del atlas. Se 


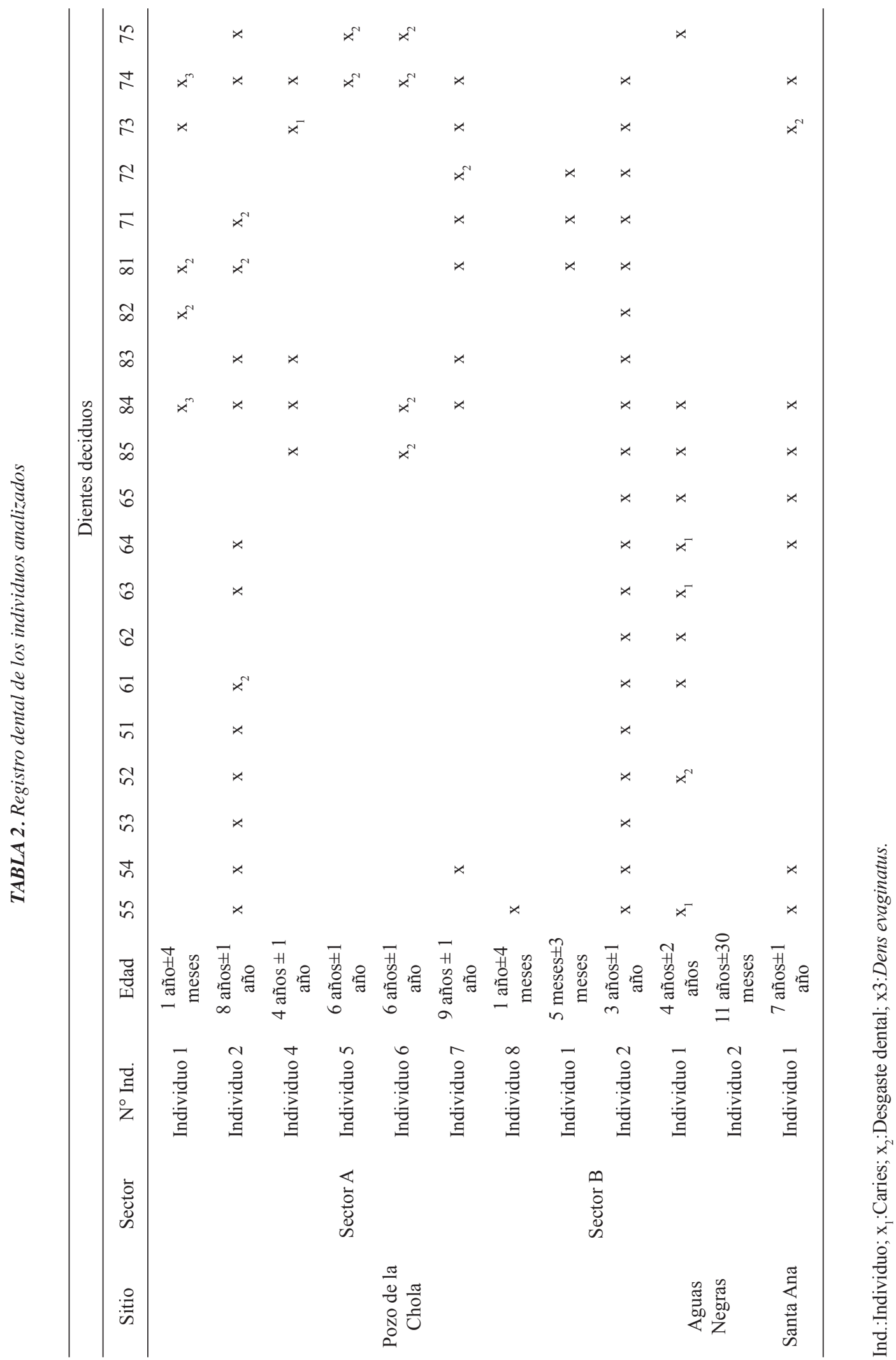


ESTUDIO DE SUBADULTOS, VALLE DE SAN FRANCISCO (0-500 DC)

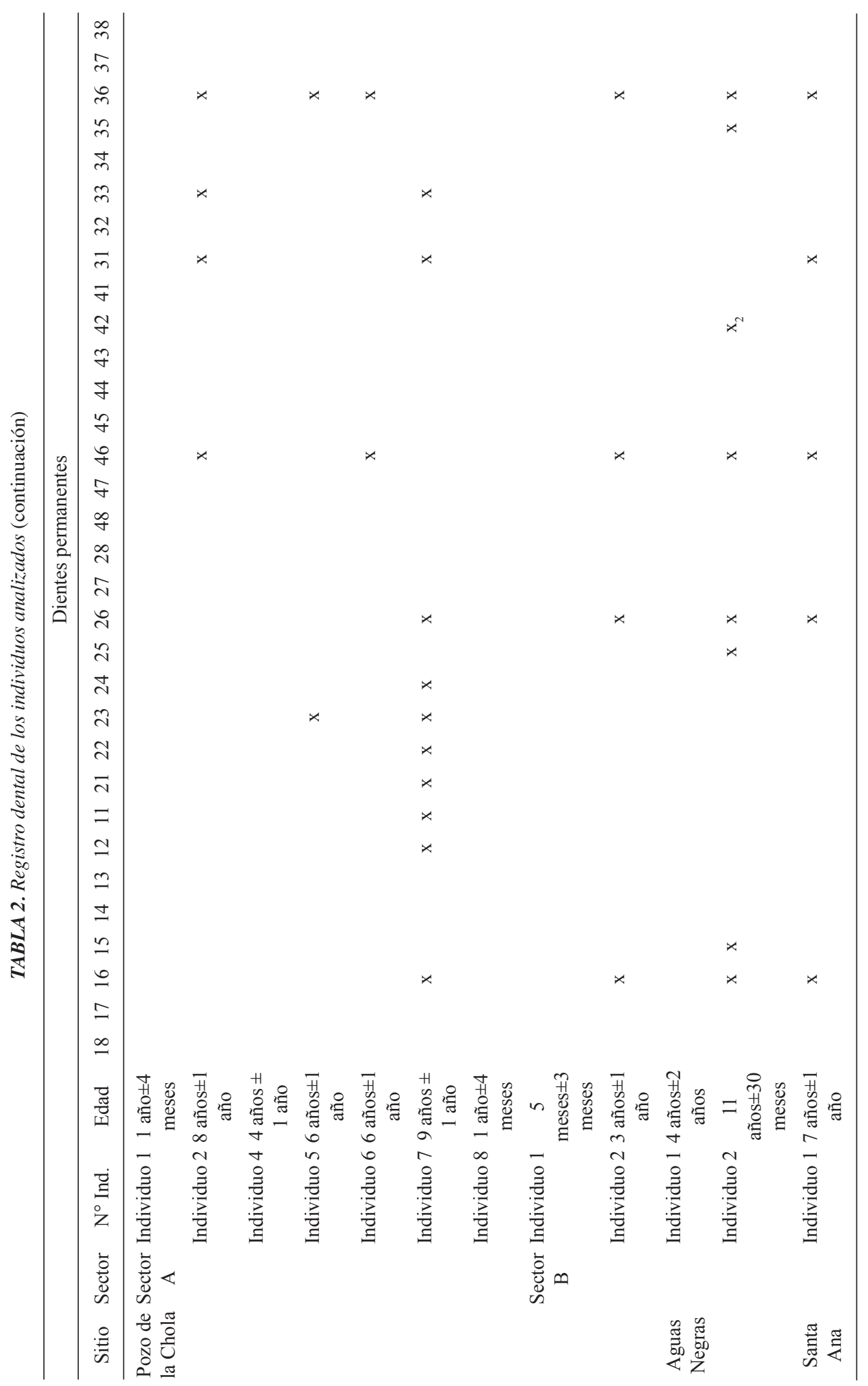

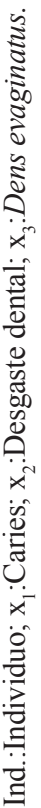



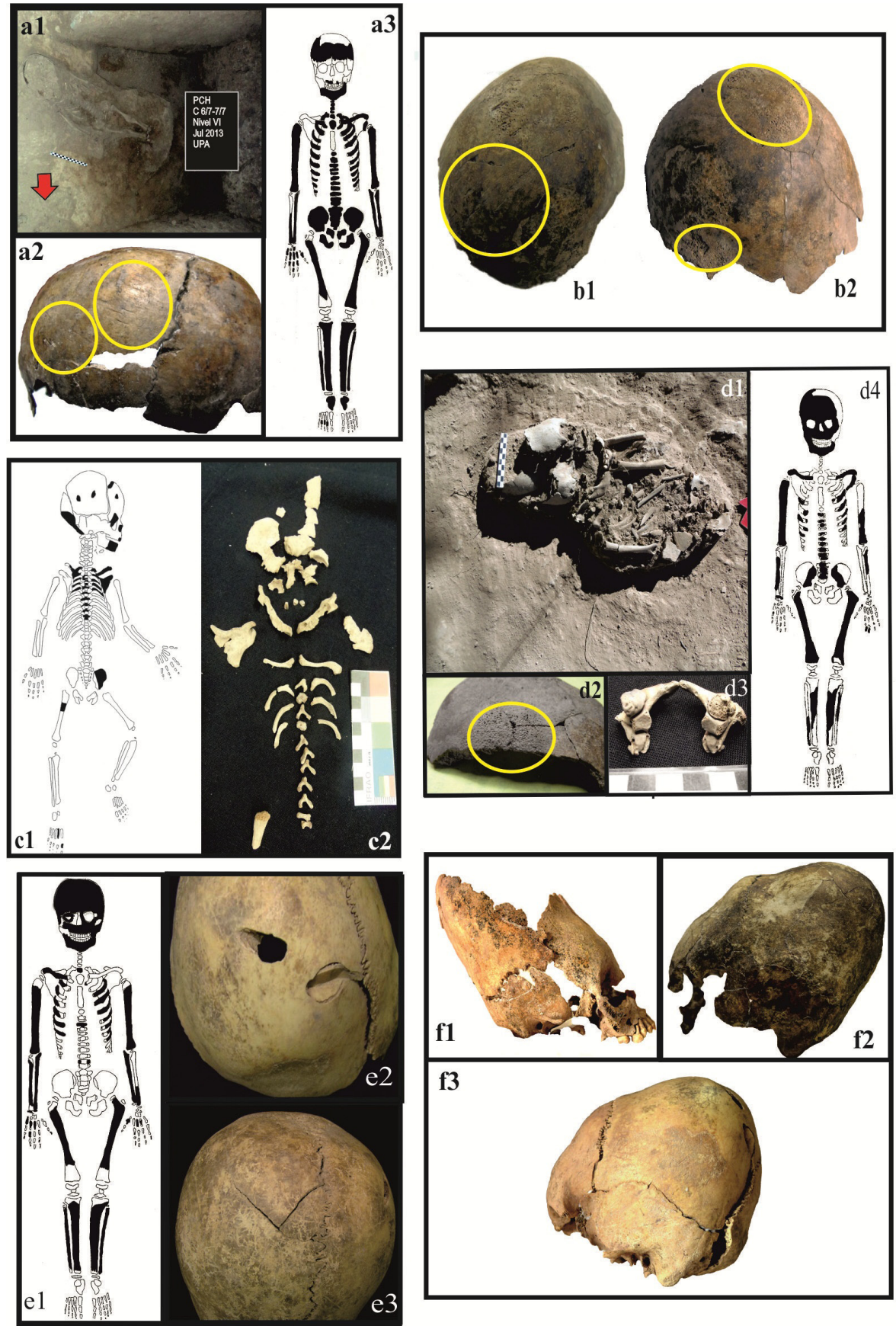

Fig. 2. 2a1. Posición de entierro del individuo 1 ( $\mathrm{PCH}$ sector A); 2a2. Elementos anatómicos recuperados, $2 \mathrm{a} 3$. Los círculos en la imagen de la izquierda indican las marcas de corte en el cráneo; $2 \mathrm{~b}$. Calota con evidencia de termoalteración, 2b1. El círculo indica la localización de las marcas de corte; 2b2. Ambos círculos indican los sectores con hiperostosis porótica; 2c. Individuo 1 (PCH sector B), 2c1. Elementos anatómicos recuperados, 2c2. Dientes con termoalteración; 2d1. Posición de entierro del individuo 2 ( $\mathrm{PCH}$ sector $\mathrm{B}$ ), 2d2. Región con hiperostosis porótica, 2d3. Fusión atlanto-occipital; 2d4. Elementos anatómicos recuperados; 2e: individuo 1 de SA; 2e1. Elementos anatómicos recuperados; 2e2. Lesiones perimortem; 2e3. Lesión post mortem; 2f1. Cráneo con deformación anular erecta (Ind. 2PCH sector B); 2f2. Cráneo con deformación tabular erecta (Ind. 1 AN); 2 f3. Cráneo con deformación tabular erecta (Ind. SA). 
observaron numerosas marcas de corte en el cráneo. En el parietal derecho, un agrupamiento de 7 huellas de corte ubicadas en la región de la eminencia parietal, las demás se encuentran dispersas en toda la superficie del hueso, sumando un total de 13. En el parietal izquierdo, se presenta un agrupamiento de 5 huellas de corte en la región de la eminencia parietal, las demás se encuentran cercanas al borde sagital y dispersas por el resto de la superficie, sumando un total de 14 (Figura 2a3). Se observaron manchas discontinuas de color marrón a negro en diferentes regiones del esqueleto pero sobre todo en la región frontal como resultado de la termoalteración. Presenta deformación cultural del cráneo a juzgar por el aplanamiento del occipital, pero no se pudo establecer la forma debido a la ausencia del resto de los huesos craneales.

Individuo 2: se trata de un entierro primario en fosa, colocado en posición decúbito dorsal extendido que fue excavado sobre el borde de la terraza del río. Presenta desgaste dental de tipo leve y ninguna carie (Tabla 2). No se registraron patologías ni marcas de corte. Por estar el cráneo incompleto solo se registró la presencia de oscículo lambdoide y foramen mental (Tabla 1). El índice de conservación es de 30,21\%, y el estado de preservación bueno. Se observan evidencias de termo-alteración en algunos sectores del esqueleto post-craneal y en los dientes, caracterizadas por un color marrón leve. Presenta deformación craneana cultural sobre la base del aplanamiento del occipital, pero no pudo determinarse la modalidad por estar ausente el resto de los huesos craneales.

Individuo 3: representado por una calota (huesos parietales) totalmente quemada con un pronunciado color que oscila entre el marrón oscuro a negro. Se encontró dentro del sedimento que rodeaba al entierro de un adulto masculino. Presenta 11 marcas de corte de pequeño tamaño en el parietal izquierdo, dispersas en toda la superficie y paralelas entre sí, con una misma orientación (Fig.2b1). Se observa hiperostosis porótica en la eminencia parietal y en la región del reborde temporal (Fig.2b2).

Individuo 4: corresponde a los restos de una calota, que fue colocada por debajo de un húmero de adulto a la manera de un "paquete". Se trataría de un entierro de tipo secundario, pero no fue posible identificar la presencia de algún pozo o disturbación estratigráfica que permita afirmar si se trata efectivamente de un evento de entierro. Presenta hiperostosis porótica en la región parietal. Tanto la calota como el hueso largo, muestran señales de termoalteración evidenciada por el color dispar que va desde un marrón leve a oscuro con pequeñas manchas discontinuas de color negro. En el mismo nivel, a una distancia menor a $0,50 \mathrm{~m}$, se recuperaron otros fragmentos de calota, una mandíbula y un fragmento de escápula. El remontaje en el laboratorio permitió corroborar que ambos hallazgos correspondían a un único individuo sub-adulto. El cráneo presenta evidencia de deformación cultural pero no pudo definirse la modalidad por estar parcialmente incompleto.

Otros hallazgos de restos humanos fueron realizados en el interior de un gran fogón en "cubeta". Se trata de restos incompletos de un individuo adulto y dos sub-adultos (individuos $5 \mathrm{y}$ 6). Un fechado realizado a una muestra de carbón del nivel $\mathrm{V}$ asociado a los restos óseos arrojó una edad de 2030 \pm 50 AP (Ortiz y Nieva, 2014a).

Individuo 5: se trata de una porción de cuerpo mandibular derecho que conserva el $1^{\circ}, 2^{\circ}$ y $3^{\circ}$ molares derechos desiduos y un canino derecho sin erupcionar. Se observa desgaste dental de tipo leve (Tabla 2). Presenta evidencia de termoalteración sobre la base del color oscuro que afectó incluso al $2^{\circ}$ molar permanente sin erupcionar.

Individuo 6: representado por 5 fragmentos de calota y una mandíbula termo-alterada que conserva $1^{\circ}$ y $2^{\circ}$ molares desiduos izquierdo y derecho (Tabla 2).

Individuo 7: corresponde a restos incompletos recuperados sobre un piso sin evidencia de entierro definitivo. Cuantificado sobre la base de 1 fragmento de malar, 1 fragmento de hueso frontal, 3 fragmentos de calota y 18 dientes, sin carie (ver Tabla 2).

Individuo 8: Se trata de restos incompletos recuperados sobre un piso sin evidencia de entierro definitivo. Representado por 9 diferentes fragmentos de distintas secciones del esqueleto: 3 fragmentos de neurocráneo, (escamas parietales derecha e izquierda); 1 fragmento de cuerpo mandibular derecho; 1 fragmento de porción petrosa o peñazco izquierdo; 1 fragmento de temporal izquierdo; 1 fragmento de epífisis proximal de tibia izquierda; 1 fragmento epífisis distal de clavícula izquierda; 1 porción izquierda de arco neural de vértebra torácica; 1 porción 
derecha de arco neural de vértebra cervical; 1 porción izquierda de arco neural de vértebra cervical; 1 fragmento de ilíaco (ilium) izquierdo; 1 fragmento óseo indeterminado; y 1 diente (Tabla 2).

\section{Sector $B$}

Individuo 1: Se encontró espacialmente muy próximo al entierro de un individuo adulto masculino. No pudo determinarse la posición de entierro porque se localizó sobre el perfil de la terraza del río, por lo que desconocemos si los huesos ausentes pudieron haberse perdido al erosionarse la terraza. El índice de conservación es de $11,03 \%$. En la Figura 2c1, se representan los elementos anatómicos recuperados. La mandíbula no se encuentra aún fusionada. Los incisivos muestran una mineralización completa de la corona. Presenta evidencia de termo-alteración en los dientes distinguible por el color marrón oscuro (Tabla 2 y Fig.2c2).

Individuo 2: se trata de un entierro primario en fosa en estrecha proximidad espacial con un individuo adulto femenino. Fue colocado en una posición fuertemente flectada y con la cara orientada hacia el adulto (Fig. 2d1). En relación a la salud dental no se observaron caries (Tabla 2). Se observa hiperostosis porótica en la región del frontal, en el reborde parietal (Fig.2d2). Presenta fusión de los cóndilos occipitales con las masas laterales del atlas (Fig.2d3). En cuanto a los marcadores no métricos se registraron: sutura metópica abierta, foramen supraorbitario, escotadura supraorbitaria, forámenes infraorbitales múltiples, osículo lambdoide, foramen mastoide, foramen mental, puente mylohioideo y apertura septal (Tabla 1). El índice de completitud es de 23,68\%.

En la Figura 2d4, se representan los elementos anatómicos recuperados. Tiene deformación craneal de tipo anular erecta (Fig. 2f1).

Los individuos 3, 4 y 5 fueron identificados a partir de 1 o 2 elementos óseos. El Individuo 5 corresponde a un fragmento de hueso largo con evidencia de alteraciones tafonómicas que se encontró incluido en el entierro de un adulto masculino, entre el hueso ilíaco y la duodécima costilla del lado derecho de este último.

\section{Aguas Negras}

El sitio se encuentra ubicado en el departa- mento Santa Bárbara, a la altura de la localidad de El Piquete, y a unos 20km aproximadamente del piedemonte de la serranía de Santa Bárbara (Fig. 1). Se recuperaron un individuo completo y restos de otro en superficie (Seldes y Ortiz, 2009).

Individuo 1: se trata de un entierro primario en fosa. El sedimento asociado al esqueleto presentaba cenizas y espículas de carbón. El individuo fue colocado en posición decúbito lateral derecho, con el pie derecho rotado por dislocación a partir de la articulación astrágalocalcánea en una posición no anatómica. Tres dientes presentan caries (Tabla 2). No se registraron patologías. En relación a los marcadores no métricos se observaron escotadura supraorbitaria, foramen mastoide y forma de la articulación astragalina en el calcáneo. El porcentaje de completitud es de 46,5\%. Presenta una coloración que va del marrón oscuro a manchas discontinuas de color negro en distintos sectores siendo más pronunciada en el cráneo como evidencia de termoalteración. Los valores de isótopos para este individuo indican una dieta vinculada probablemente al maíz y las crasuláceas $\left(\boldsymbol{\delta}^{13} \mathrm{C}_{\mathrm{co}}-10,72\right.$ y $\left.\delta^{15} \mathrm{~N} 7,74\right)$ (Ortiz y Killian Galván, 2016). El fechado sobre hueso arrojó una edad de 1650 \pm 80 años AP (Ortiz et al., 2015) (Tabla 1). Presenta deformación craneana de tipo tabular erecta (Fig. 3f2).

Individuo 2: se encuentra representado solamente por una mandíbula. Los dientes no presentan caries, pero sí desgaste del esmalte de tipo leve (Tabla 2) (Seldes y Ortiz, 2009).

\section{Arroyo Colorado}

Ubicado en el departamento Santa Bárbara, el sitio arqueológico se encuentra emplazado en el interior de una finca privada y los restos óseos fueron exhumados por particulares durante un rescate (Tabla 1 y Fig. 1).

Individuo 1: corresponde a un individuo sobre la base de mediciones morfométricas (omóplato completo y mandíbula).

\section{Santa Ana}

El sitio se encuentra ubicado en el casco urbano de la ciudad de San Pedro, barrio Santa Ana (Fig. 1). En el año 2009, Policía de la provincia de Jujuy, exhumaron los restos de un sub-adulto 
luego de la denuncia de su hallazgo por parte del personal encargado de la instalación de red de gas domiciliaria. Se observa desgaste dental de tipo leve, sin caries (Tabla 2), y ninguna patología de estrés metabólico. En relación a los marcadores no métricos se registraron: foramen supraorbitario, hueso apical, oscículo lambdoide, oscículo asterion, foramen mastoide, foramen mental y apertura septal (Tabla 1). El índice de conservación es de 29,72\%. En la Figura 2e1, se representan los elementos anatómicos recuperados. Presenta lesiones traumáticas en el cráneo; dos lesiones perimortem a nivel de la mitad posterior del parietal izquierdo (Fig. 2e2), y una postmortem en el tercio medio del parietal derecho (Fig. 2e3). La información paleodietaria para este individuo arroja valores $\boldsymbol{\delta}^{13} \mathrm{C}$ más empobrecidos $(-14,9)$, los cuales pueden ser vinculados con los recursos acuíferos y vegetales con vía fotosintética C3 (Ortiz y Killian Galván, 2016). Presenta deformación cultural del cráneo de tipo tabular erecta (Fig. 2f3).

\section{RESULTADOS Y DISCUSION}

A partir del análisis de los sub-adultos del valle de San Francisco, disponemos de una serie de indicadores que, considerando el contexto ambiental y la cronología, pueden ayudarnos a entender las condiciones de vida donde se desarrollaron las poblaciones bajo estudio, así como aquellas prácticas vinculadas al tratamiento funerario.

Como se mencionó en la primera sección del trabajo, la mayor cantidad de datos proviene del sitio Pozo de la Chola (PCH) con un número de 11 individuos. Otros 4 fueron recuperados de diferentes sitios (Tabla 1). La población de subadultos representa en $\mathrm{PCH}$ el $28 \%$ de la muestra. Aunque la cantidad de individuos es escasa para cualquier inferencia de carácter estadístico, si la comparamos con el porcentaje de adultos exhumados del mismo sitio (72\%), observamos que la mortalidad podría ser considerada elevada. Sobre el total de individuos sub-adultos que incluye a los recuperados en los 4 sitios arqueológicos, a 13 se les pudo estimar la edad de muerte (Tabla 1). De acuerdo a esta información observamos que las edades abarcan casi todas las franjas etarias, estando mayormente representada la de los niños (46\%), seguidos por los infantiles (31\%). Aunque esta situación responde al modelo demográfico propio de poblacio- nes antiguas con elevados índices de mortalidad en sub-adultos, el mayor número corresponde a la franja etaria por encima de la edad con la más alta tasa de mortalidad (primer mes de vida hasta el $2^{\circ}$ año), si consideramos también a los juveniles (23\%) (Lewis, 2007).

Desde el punto de vista del estado de salud, se identificaron 3 individuos con hiperostosis porótica. Uno de ellos (que corresponde al que presenta mejor preservación), no exhibe ninguna otra evidencia de patologías relacionadas a episodios de estrés metabólico. En los otros dos casos, por tratarse solamente de fragmentos craneales, no puede estimarse la presencia de alguna otra señal de enfermedades crónicas. El resto no ofrece evidencias de patologías vinculadas con episodios de estrés metabólico. La edad dental y la basada en los métodos morfométricos, resultaron en una notoria concordancia, lo que permite proponer que los individuos no habrían estado expuestos a factores retardantes del crecimiento. Respecto de la hiperostosis, diversos autores han podido identificar etiologías muy distintas (Harb y Martinez, 2004; Huicochea Gomez, 2003; Walker et al., 2009, entre otros). Se trata de un indicador esqueletal poco específico que no permite realizar interpretaciones certeras si es tomado en forma aislada. Parece estar vinculada con anemias de tipo megaloblásticas debido a la carencia de vitamina B12 y B9 (ácido fólico). Si tenemos en cuenta los datos del registro arqueofaunístico, la deficiencia de vitamina B12 por dietas inadecuadas y carentes de alimentos de origen animal, no sería una explicación plausible. Otras etiologías podrían ser la causa primaria de las hiperostosis observadas. Las hipótesis suscitadas por los registros multi proxis de Pozo de la Chola (análisis antracológicos; de fitolitos; polínicos y de arqueofauna), indican que el ambiente era muy similar al actual. Los climas tropicales, como ya se mencionó, suelen propiciar la existencia de vectores trasmisores de diferentes enfermedades, por ejemplo, diversos tipos de parásitos. En Pozo de la Chola, hospedadores parasitarios como los caracoles de la familia Ampulariidae, parecen haber formado parte de la dieta (Ortiz y Vargas Rodríguez, 2015). Las parasitosis abarcan un amplio rango de parásitos y hospedadores, pueden o no ser de alta morbilidad y transmisibles vía transplacentaria, llegan a ser asintomáticas por un tiempo, y de acuerdo al ór- 
gano afectado, dependerá el cuadro clínico. La gravedad de las infecciones depende del tipo de parásito, la vía de infección y la respuesta inmunitaria del hospedador. Sin embargo en los subadultos, cualquier infección parasitaria puede provocar anomalías en el crecimiento y en el desarrollo mental, lo que causa, además, anemia y desnutrición (Becerril, 2011). Los casos observados podrían evidenciar un cuadro de deficiencia de absorción intestinal a causa de las parasitosis, lo que habría redundado en anemia.

Otra patología observada corresponde a dos casos de fusión atlanto-occipital registrada en individuos de Pozo de la Chola. Se trata de una importante malformación congénita de la región cráneo-vertebral debido a la proximidad a la región raquimedular. La acción de los músculos posturales sobre la superficie extensora puede ser afectada por esta anomalía y puede producir un amplio rango de signos y síntomas neurológicos, que varían desde un dolor de cabeza transitorio a un síndrome neurológico agudo (Campos et al., 2012). Es importante destacar que la incidencia de esta malformación varía entre $0.14 \%$ y $0.75 \%$, a nivel de la población mundial (Monalisa, Pandit y Bhatnagar, 2017). Resulta muy llamativa entonces su prevalencia en dos individuos del mismo sitio.

La presencia de dens evaginatus en el individuo 1 del sector A, marca la existencia de una patología de herencia autosómica, ya que aunque aún se discute su etiología, se ha observado que responde a patrones de herencia ligada al cromosoma X (Chávez Ovalle y Mendiola Aquino, 2011). La incidencia familiar ha sido descrita y se ha sugerido un componente hereditario en su etiología. Se trata del infantil con occipitalización del atlas (Tabla 1), por lo cual es importante destacar la presencia en un mismo individuo de dos patologías muy infrecuentes en la población mundial, y ambas consideradas como de trasmisión hereditaria.

En lo que respecta a los marcadores no métricos, fueron relevados en 5 individuos. Aunque su frecuencia no permite establecer por el momento ninguna estimación de distancia poblacional, su registro puede ser utilizado para realizar en el futuro comparaciones intra e inter poblacionales, considerando que numerosos marcadores epigenéticos muestran variaciones regionales distintivas (Sutter y Metz, 2004).
En relación a la salud dental, se observa que la presencia de caries es relativamente baja, y se ha detectado desgaste dental de tipo leve en 5 individuos con edades que abarcan desde 1 hasta los 9 años. Aunque las dietas en sociedades prehispánicas incluían alimentos con un componente abrasivo debido a las condiciones de procesamiento y preparación, es llamativo el desgaste observado en los individuos de menor edad, puesto que la atrición dental es un fenómeno que se incrementa con la actividad masticatoria a lo largo de los años (Hillson, 1996), y por lo tanto no esperable en individuos muy jóvenes.

Con respecto a las prácticas mortuorias, de los 4 niños completos procedentes de sepulturas excavadas por nosotros, ha sido posible determinar la posición de entierro. Dos fueron colocados en decúbito dorsal extendido y uno en posición flectada decúbito lateral izquierdo, lo que implica que tuvo que ser atado o enfardado para que conservara una posición tan comprimida. El individuo de Aguas Negras fue colocado decúbito lateral flexionado. En consecuencia, no ha sido posible apreciar diferencias relevantes en la posición de entierro de los sub-adultos de acuerdo a la edad, pero no podemos establecer si el sexo fue un parámetro a considerar. Otra clase de evidencias sugiere prácticas vinculadas a la manipulación de los cuerpos peri y/o postmortem. Entre ellas, la exposición al fuego. Un elevado porcentaje de los sub-adultos presenta señales de termoalteración (60\%) (Tabla 1). En dos casos ha sido posible relevar que el evento de quema se produjo en la misma fosa donde fueron inhumados. En el caso del individuo 1 del sector A, 9 taxa arbóreas fueron utilizadas como leña sobre la base del análisis antracológico de los carbones recolectados en la fosa de inhumación (Ortiz et al., 2017). Un dato adicional vinculado a la disposición del cuerpo del entierro de Aguas Negras, se refiere a la posición irregular de la flexión anatómica del pie derecho. Este se presenta rotado hacia atrás por acción mecánica perimortem, no patológica, ya que la morfología del hueso es normal y no presenta anomalías estructurales, mal formaciones, ni fracturas. Se descarta como causa, la exposición al fuego, ya que la flexión y abducción ocurren sobre las articulaciones de hombro, codo, cadera y rodillas (Holck, 2008). Además, en un cuerpo muerto la capacidad de contraerse 
decrece notablemente, y la posición irregular se presenta unilateralmente en este caso. Todo ello nos lleva a suponer algún tipo de gesto vinculado al ritual funerario cuyo significado no podemos discernir.

Dos fechados radiocarbónicos realizados a individuos sub-adultos, así como otros dos de adultos en Pozo de la Chola, indican episodios tanto diacrónicos como sincrónicos de entierro (Ortiz y Nieva, 2014b). Los más cercanos en el tiempo, muestran que la forma de enterrar a los sub-adultos no difería de la practicada con los adultos. En ambos casos, el entierro primario directo en fosa fue el más común, y fueron generalmente enterrados sin acompañamiento mortuorio, al menos en lo que se refiere a bienes no perecederos. La mayor cantidad de elementos interpretados como de carácter intencional acompañando a entierros de su-adultos, han sido reportados por la expedición sueca para los niños del cementerio de urnas (Boman, 1908; Nordenskiöld, 1903). Sin embargo, es interesante mencionar, que hasta el momento son los únicos que presentan como acompañamiento mortuorio objetos locales y foráneos (gasterópodos del pacífico y cuentas discoidales de aragonita). A diferencia de los adultos con piezas que remiten a la comensalidad o el consumo individual (escudillas de cerámica y pipas) (Ortiz y Nieva, 2014b), los elementos del mundo funerario de los niños se asocian a animales (diversos tipos de caracoles y especialmente gasterópodos), o en su defecto, cuentas de collar líticas. Sin embargo, desconocemos si en los entierros en urnas como en los primarios, fueron colocados elementos perecederos como acompañamiento mortuorio. Un dato de interés reviste el resultado del análisis de fitolitos de una muestra de sedimento del entierro del sub-adulto 2 del sector B de PCH. Fue colectada a la altura del esternón y presentó fitolitos cavados afines a hojas de cucurbitáceas, siendo la única, de las 4 procesadas vinculadas con diferentes entierros, y de las 15 recolectadas en diversos sectores del sitio arqueológico; con la presencia de esta clase de micro-restos (Zucol, 2015). Si bien su existencia puede obedecer a una incorporación accidental (al rellenarse el pozo luego de la depositación del cuerpo), otra alternativa válida puede ser, que se colocaran restos vegetales como parte de la preparación de la tumba o del ritual funerario.
Los sub-adultos también fueron objeto de intervenciones perimortem registradas a partir de marcas de corte. En dos oportunidades se observaron marcas en los parietales, acción que posiblemente estuvo vinculada con la extracción del cuero cabelludo o el descarne del cráneo (Spencer, 2007). Debido a que el color de la sección transversal de las huellas es idéntico al de la superficie del hueso, se puede postular que este procedimiento fue realizado antes de que el cuerpo fuera sometido a la acción del fuego.

Los datos que nos aporta el registro arqueológico muestran que el mismo procedimiento se habría efectuado a adultos y sub-adultos (Ortiz y Nieva, 2014a) y en Pozo de la Chola, además, ambos comparten el mismo espacio funerario. Esto podría indicar que los niños tenían reconocimiento social como individuos que participan de una identidad comunitaria y a la vez específica, en una estructura social aparentemente simétrica. Al ser enterrados del mismo modo que los adultos pueden estar reflejando concepciones acerca de la edad social, donde no se observa una distinción de acuerdo a la clase etaria de los inhumados. Sin embargo tenemos otros casos, con presencia de algunos huesos desarticulados dispersos en los pisos o encontrados asociados al entierro de adultos masculinos. Los restos óseos aislados han sido registrados en otros sitios de la región y se deben a acciones intencionales. Así, aunque los huesos aislados del sector B podrían deberse a eventos de disturbación postdepositacionales, no podemos descartar que su disposición sea intencional, considerando la evidencia del sector A, donde hemos realizado hallazgos de partes anatómicas seleccionadas y dispuestas en diferentes contextos que incluyen adultos y sub-adultos. Los restos óseos recuperados en el fogón en cubeta, corresponden a dos niños y un adulto. En otros casos, individuos representados solamente por algunos elementos anatómicos, especialmente del cráneo, se encontraron depositados sobre los pisos de ocupación, y en un caso, se conformó una especie de "paquete" compuesto por huesos de un niño (cráneo) y de un individuo adulto (fémur). Además, existe el dato del cementerio de párvulos (Boman, 1908; Nordenskiöld, 1903), lo que indica un escenario en donde algunos sub-adultos fueron segregados del espacio residencial e inhumados en un lugar funerario exclusivo y acompañados por 
elementos materiales a la manera de acompañamiento mortuorio.

A pesar del cuidado, que parecen haber recibido, no estuvieron exentos de situaciones de violencia. El traumatismo craneal documentado en el juvenil de Santa Ana, plantea una situación de violencia interpersonal. Los traumatismo se encuentran en la zona posterior del cráneo, más común en situaciones de ataque que de enfrentamientos frontales (Rihuete Herrada et al., 2011).

Hasta el momento hemos registrado dos modalidades de deformación del cráneo en los subadultos (tabular erecta y anular erecta). A juzgar por el aplanamiento del occipital registrado en otros cráneos incompletos, sugerimos que la deformación craneana debió ser una práctica generalizada en toda la población. Sin embargo, por el momento, no podemos vincular las variedades deformatorias con alguna clase de parámetro específico, por ejemplo el sexo. La costumbre de modificar la forma del cráneo, fue una práctica frecuente en el NOA, sin embargo la modalidad anular erecta no se menciona para la región de las selvas occidentales siendo considerada inexistente (Cocilovo y Varela, 2010). Esta información, conjuntamente con la recabada en relación a las costumbres inhumatorias, parece marcar prácticas idiosincráticas distintivas. Por ejemplo, la combinación de intervención peri y/o postmortem (scalping-descarne) y exposición al fuego, acompañado de entierro primario directo, se presenta hasta el momento como una característica exclusiva para las poblaciones subandinas de Jujuy. A su vez, la información recabada en relación a patologías y perfiles etarios, nos permite conocer la calidad medioambiental postnatal (que incluye tanto factores físicos como humanos), y avanzar en el conocimiento sobre la condiciones de reproducción biológica que imperaron en la región durante el Formativo temprano. Con el avance de los trabajos y la incorporación de nuevos datos, se espera poder evaluar las hipótesis formuladas sobre distancia poblacional, situaciones de violencia interpersonal y el rol que ocuparon los sub-adultos en el seno de estas sociedades.

\section{AGRADECIMIENTOS}

A los alumnos de la FHyCS, UNJu, que participaron en las diferentes campañas arqueo- lógicas de Pozo de la Chola, y a los dos revisores anónimos cuyos comentarios permitieron enriquecer notablemente la versión original del trabajo.

\section{LITERATURA CITADA}

Aufderheide, A. y Rodríguez Martín, C. (1998). The Cambridge enciclopedia of human paleopathology. Cambridge, Reino Unido: Cambridge University Press.

Becerril, M. A. (2011). Parasitología médica. Tercera edición. Madrid, España: McGraw-Hill Editorial.

Boman, E. (1908) [1991]. Antigüedades de la región andina de la República Argentina y del desierto de Atacama. Jujuy, Argentina: Universidad Nacional de Jujuy

Botella Lopez, M. 2005. Diagnóstico diferencial de las marcas de corte sobre los huesos humanos. En Cañelas Trobat, A. (Ed.), Nuevas perspectivas del diagnóstico diferencial en Paleopatología (pp. 87-100). Menorca, España: Asociación Española de Paleopatología.

Buikstra, J. E. y Ubelaker, D. H. (Eds.). (1994). Standards for data collection from human skeletal remains. Fayetteville, Estados Unidos: Arkansas Archaeological Survey, Research Series. No. 44.

Campillo, D. y Subirá, E. (2004). Antropología fisica para arqueólogos. Barcelona, España: Ariel.

Campos, D., Silva, T., Ellwanger, J., Goerck, M., Kipper, J., Piazza, J. y Kraether Neto L. (2012). Atlanto-occipital fusion and its neurological complications: a case report. Journal Morphological Science, 29(2), 111-113.

Chávez Ovalle, S. C. y Mendiola Aquino, C. (2011). Dens evaginatus, tratamiento de absceso apical agudo: reporte de caso. Revista de Estomatología Herediana, 21(1), 34-37. doi:10.20453/reh.v21i1.1728

Cocilovo, J. A. y Varela, H. (2010). La distribución de la deformación artificial del cráneo en el área andina centro sur. Relaciones de la Sociedad Argentina de Antropología, $X X X V, 41-68$

Dougherty, B. (1975). Nuevos aportes para el conocimiento del Complejo Arqueológico San Francisco (sector septentrional de la región de las selvas occidentales argentinas, subárea del noroeste argentino) (Tesis de doctorado). Facultad de Ciencias Naturales y Museo, Universidad Nacional de La Plata, La Plata.

Esponda Vila, R. (1994). Anatomía dental. D.F., México: Universidad Nacional Autónoma de México (UNAM).

Halcrow, S. (2015). Infants in the bioarchaeological record: Who cares? Artículo presentado en Society for the Study of Childhood in the Past. Recuperado de https:// otago.academia.edu/SianHalcrow/Papers

Halcrow, S. y Tayles, N. (2011). The bioarchaeological investigation of children and childhood. En Agarwal, S. y Glencross, B. (Eds.). Social bioarchaeology (pp. 333-360). Oxford, Reino Unido: Blackwell Publishing. doi:10.1002/9781444390537.ch12

Harb, E., J. y Martínez, N. (2004). Hiperostosis cortical postrauma. Presentación de un caso. Acta Ortopédica Mexicana, 18(4): 160-164.

HIllson, S. (1996). Dental anthropology. Cambridge, Reino Unido: Cambridge University Press.

Holck, P. (2008). Cremated bones. A medical-anthopological study of an archaeological material on cremation burial. Oslo, Noruega: University of Oslo.

Huicochea Gomez, L. (2003). Evidencia de anemia en una seria esquelética actual. Estudios de Antropología Biológica, XI, 277-295. 
Imbelloni, J. (1932). Sobre un ejemplar mimético de deformación craneana. Anales del Museo Nacional de Historia Natural, 37, 193-208.

Lewis, M. (2007). The Bioarchaeology of children. Perspectives from biological and forensic Anthropology. Cambridge, Reino Unido: University Press.

Martin, D. L., Goodman, A. y Armelagos G. (1985). Skeletal pathologies as indicators of the quality and quantity of diet. En Gilbert, R. I. y Mielke, J. H. (Eds.), The analysis of prehistoric diets (pp. 227-279). San Diego, Estados Unidos: Academic Press.

Meneghello, J., Fanta, E. y Paris, E. (1972). Pediatría. Tomo 1. Madrid, España: Editorial Médica Panamericana.

Monalisa, Pandit, S. y Bhatnagar, R. (2015). Atlanto-occipital fusion: A case report. Medical Journal of Dr. D.Y. Patil University, 8(5), 636-638. doi:10.4103/09752870.164963

Nájera Colino, T., Molina González, F., Jiménez-Brobeil, S., Sánchez Romero, M., Al Oumaoui, I., Aranda Jiménez, G., Delgado-Huertas, A. y Laffranchi, Z. (2010). La población infantil de la Motilla del Azuer: Un estudio bioarqueológico. Complutum, 21(2), 69-102.

Nordenskiöld, E. (1903) [1993]. Lugares precolombinos de asentamiento y entierro en la frontera sudoeste del Chaco. Jujuy, Argentina: Universidad Nacional de Jujuy.

Ortiz, G. (2007). La evolución del uso del espacio en las tierras bajas jujeñas (subárea del río San Francisco) (Tesis de Doctorado). Facultad de Filosofía y Humanidades, Universidad Nacional de Córdoba, Córdoba.

Ortiz, G (2014). Muerte y ancestralidad en poblaciones sub-andinas de la cuenca del San Francisco, noroeste de Argentina, En Combès, I. y Villar, D. (Eds.), Serie Scripta Autoctona (pp. 95-108). Cochabamba, Bolivia: Editorial Itinerarios-Centro de Investigaciones Históricas y Antropológicas.

Ortiz, G. y Killian Galván, V. (2016). El consumo como vía para comprender economías mixtas. Su aplicación al sur del valle de San Francisco, región pedeontana de Jujuy (Argentina). En Alconini, S. (Ed.), Las montañas tropicales y valles contiguos de los andes sud-centrales (pp. 263-282). Bolivia.

Ortiz, G y Nieva, L. (2014a). Morir en el valle de San Francisco. Prácticas funerarias, termo-alteración y estrategias de memorización en la selva pedemontana de las yungas del NOA. Zeitschrift für Archäologie Aussereuropäischer Kulturen 6, 247-276.

Ortiz, G. y Nieva, L. (2014b). Rituales y memorias del pasado. Practicas funerarias en la región del rio San Francisco, Jujuy, Argentina. En Seldes, V. y Gheggi, M. S. (Eds.), Antropología Biológica y estudios del comportamiento mortuorio de los pueblos prehispánicos del Noroeste Argentino (pp. 149-174). Buenos Aires, Argentina: Fundación de Historia Natural Félix de Azara.

Ortiz, G. y Vargas Rodríguez, N. (2015). Más allá del artefacto. Aproximación al ambiente y estrategias de uso de moluscos en la región del Valle de San Francisco, Jujuy (0 al 500 dc). En Hammond, H y Zubimendi, M. A. (Eds.), Arqueomalacología. Abordajes metodológicos y casos de estudio en el Cono Sur (pp. 59-78). La Plata, Argentina: Fundación de Historia Natural Félix de Azara.

Ortiz, G., Heit, C., Nieva, L., Zamora, F., Batallanos, N. y Chapur, F. (2015). Pensando al formativo desde la región pedemontana de las yungas de Jujuy. En Korstanje, A., Lazzari, M., Basile, M., Bugliani, F., Lema, V., Pereyra Domingorena, L. y Quesada, M. (Eds.), Cróni- cas materiales precolombinas. Arqueología de los primeros poblados del Noroeste Argentino (pp. 695-720). Buenos Aires, Argentina, SAA.

Ortiz, G., Ramos, R. S. y Alavar, A. (2017). Fire, rituals and domesticity. Forest resource management in the subAndean region of Jujuy, Argentina (2000 BP): First anthracological evidence. Journal of Anthropological Archaeology, 47, 96-108. doi:10.1016/j.jaa.2017.04.002

Ortiz, G., Seldes, V. y Bosio, L. (2012). Evidencias de trauma en el periodo formativo en el valle de San Francisco, Jujuy, Argentina. Archaeobios, 6(1), 33-52.

Pijoan, C. M., y Pastrana, A. (1987). Método para el registro de marcas de corte en huesos humanos. El caso Tlaltelcomila, Tetelpan, D.F. En Carmona, M. (Coord.), El Preclásico o Formativo. Avances y perspectivas (pp. 419-436). D.F., México: MNA-INAH.

Pijoan, C. M., Mansilla, J., Leboreiro, I. V., Lara, V. y Bosch, P. (2008). Thermal alterations in archaeological bones. Archaeometry, 49, 713-727. doi:10.1111/j.14754754.2007.00331.x

Rihuete Herrada, C., Oliart Caravatti, C. y Fregeiro Morador, M. I. (2011). Aproximación a la población Argárica a la luz de los enterramientos del convento de Madres Mercedarias de Lorca (Murcia). Alberca, 9, 39-79.

Rumi, A. y Nuñez, V. (2013). Gasterópodos continentales de importancia sanitaria en el noroeste de Argentina. En Salomón O. D. A. Rumi, A. (Eds.). Moluscos de interés sanitario en Argentina (pp. 7-39). Puerto Iguazú, Argentina: INMET.

Sanchez Romero, M. (2010). ¡Eso no se toca! Infancia y cultura material en arqueología. Complutum, 21(2), 9-13.

Seldes, V. y Ortiz, G. (2009). Avances en los estudios bioarqueólogicos de la región del río San Francisco, Jujuy, Argentina. Andes, 20, 15 - 35.

Scheuer, L. y Black, S. (2000). Developmental juvenile osteology. San Diego, Estados Unidos: Academic Press

Solari Giachino, A. (2010). Identificación de huellas de manipulación intencional en restos óseos humanos de origen arqueológico (Tesis de doctorado). Universidad de Granada, Granada.

Spencer S. (2007). Cutmarks on crania at the late Mississippian Mann site cemetery, Posey County, Indiana. Artículo presentado en Midwest Archaeological Conference (MAC), Notre Dame, South Bend, Recuperado de http://www.academia.edu/1717906.

Stodder, A. L. (2008). Taphonomy and the nature of archaeological assemblages. En Katzenberg, M. A. y Saunders, S. R. (Eds.), Biological anthropology of the human skeleton (pp. 71-114). New Jersey, Estados Unidos: Editorial Wiley-Liss. doi:10.1002/9780470245842.ch3

Sutter, R. y Metz, L. (2004). Nonmetric cranial trait variation and prehistoric biocultural change in the Azapa valley, Chile. American Journal of Physical Anthropology, 123, 130-145. doi:10.1002/ajpa.10311

Ubelaker, D. (1999). Human skeletal remains, excavation, analysis, interpretation. Chicago, Estados Unidos: Aldine Pub.

Walker, P. L., Bathurst, R. R., Richman, R., Gjerdrum, T. y Andrushko, V. (2009). The causes of porotic hyperostosis and cribra orbitalia: a reappraisal of the irondeficiency-anemia hypothesis. American Journal of Physical Anthropology, 139, 109-125. doi:10.1002/ ajpa. 21031

Zucol, A. (2015). Análisis de presencia de microrestos en muestras de la localidad arqueológica Pozo de la Chola, Jujuy, Argentina. Informes del Laboratorio de Paleobotánica 39 (CICYTTP-Diamante). 